\title{
Causal Pathway to Health-Related Quality of Life in People with Psychotic Disorders: the Role of Loneliness and its Contributors
}

\begin{abstract}
A.G. Nevarez-Flores ${ }^{1}$, M. Breslin'1, V. Carr², V.A. Morgan ${ }^{3,1}$, A. Waterreus 3 , C. Harvey ${ }^{4}$, K. Sanderson ${ }^{5,1}$, A.L. Neil' ${ }^{1}$.

1 University of Tasmania, Menzies Institute for Medical Research, Hobart, Australia.

2University of New South Wales, Research Unit for Schizophrenia Epidemiology- School of Psychiatry, Sydney, Australia.

${ }^{3}$ The University of Western Australia, Neuropsychiatric Epidemiology Research Unit, School of Population and Global Health, Crawley,

Australia.

${ }^{4}$ The University of Melbourne, Psychosocial Research Centre- Department of Psychiatry, Melbourne, Australia.

5 University of East Anglia, School of Health Sciences, Norwich, United Kingdom.
\end{abstract}

\section{Background}

Perception of loneliness has been identified as one of the top challenges for people with psychotic disorders ${ }^{1}$ as well as the strongest predictor of health-related quality of life (HRQoL) in this population² (see Abstract Number: WCP19-0388).

\section{Objectives}

1) Establish a model of contributors to the perception of loneliness in people with psychotic disorders, based on a suite of sociodemographic and clinical characteristics.

2) Ascertain the mediating role of loneliness in the relationship between identified contributors to loneliness and other known predictors of $\mathrm{HRQoL}$ with $\mathrm{HRQ}$ oL in this population.

\section{Materials and Methods}

Data were collected during the 2010 Australian National Survey of Psychosis ${ }^{1}$, and limited to individuals with an ICD-10 psychotic disorder. HRQoL was assessed with the Assessment of Quality of Life (AQoL)-4D. Perception of loneliness in the last 12-months was determined through a five-level categorical variable.

\section{Statistical analyses}

A three-stage model
building process was
undertaken:
1. Univariable
associations with
loneliness were
tested

A multiple linear
regression model for

\section{Results}

Nine of 23 variables had an independent effect on loneliness: social dysfunction, experienced stigma, contact with friends, diagnosis, service utilisation in the last 12-months, depressive symptoms, anxiety, arthritis and traumatic events in childhood. The model explained $13 \%$ of the variance of loneliness.

In the mediation analysis, ten of 16 included variables were partially mediated through loneliness (see Figure). The greatest mediation was for contact with friends then social dysfunction. Loneliness was the strongest predictor of HRQoL and the model explained $49 \%$ of the variance of HRQoL.

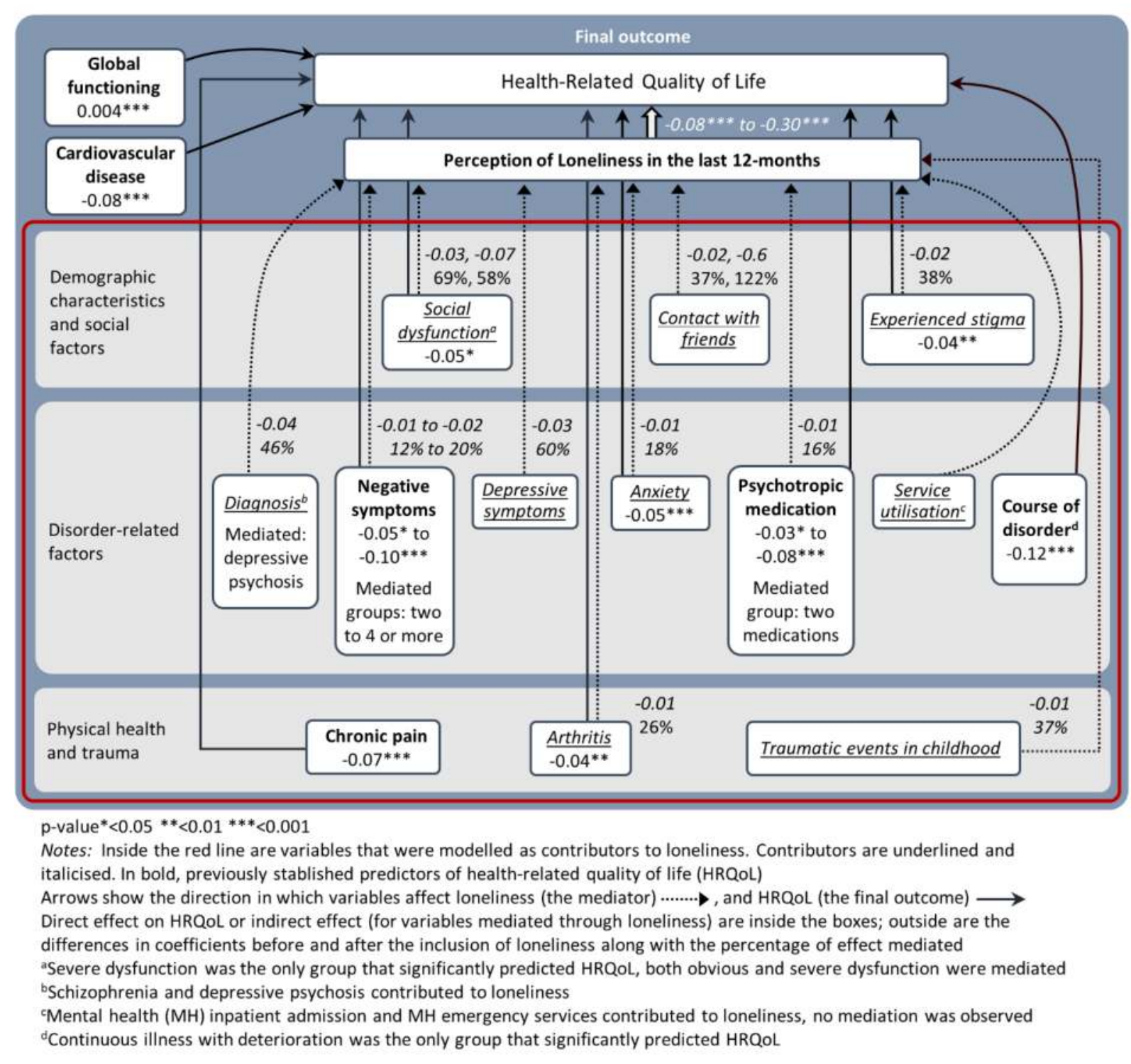

\section{Conclusions}

Contributors to loneliness and the mediating effect of loneliness in the relationship between HRQoL and contributors to loneliness and known predictors of HRQoL were ascertained. Our findings provide a focus for strategies aimed at diminishing the perception of loneliness among people living with a psychotic disorder, and in turn improving their HRQoL.

References

1. Morgan VA, Waterreus A, Jablensky A et al. People living with psychotic illness in 2010: the second Australian national survey of psychosis. ANZJP 2012; 46: 735-52.

2. Nevarez-Flores AG, Breslin M, Carr VJ et al. The Mediating Role of Global Functioning in the Relationship between Health-Related Quality of Life and Sociodemographic and Clinical Characteristics of People with Psychotic Disorders. Submitted. (WCP19-0388) 